\title{
A CHERENKOV THRESHOLD COUNTER USING LIQUID CORE FIBER FOR ESTIMATION OF RADIOACTIVE WASTE INVENTORY
}

\author{
Jun Kawarabayashi \\ Nagoya University \\ kawarabayashi@nucl.nagoya- \\ u.ac.jp
}

Hideki Tomita

Nagoya University

tomita@nagoya-u.jp
Nagoya University

satou.yousuke@f.mbox.nagoyau.ac.jp

\author{
Hikaru Hayakawa \\ Nagoya University \\ hayakawa.hikaru@d.mbox.nagoya- \\ u.ac.jp
}

Tetsuo Iguchi

Nagoya University

t-iguchi@nucl.nagoya-u.ac.jp

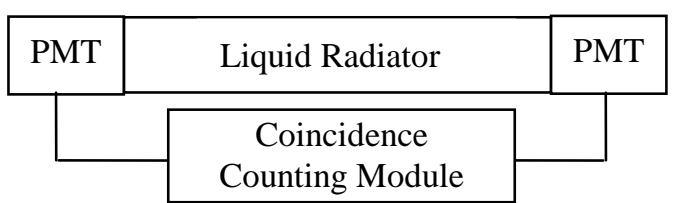

Fig. 1 Schematic view of proposed detector

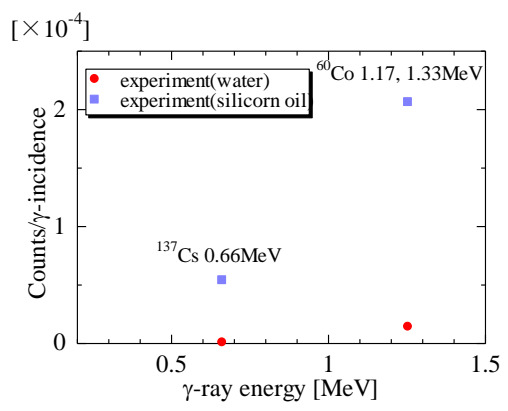

Fig. 2 Experimental results of the detection efficiency for gamma-rays of ${ }^{60} \mathrm{Co}$ and ${ }^{137} \mathrm{Cs}$.

silicone oil (KF-56 Shin-Etsu Chemical Co., Ltd.) covered by a pyrex glass tube. The center position of the detector was irradiated by gamma-rays from ${ }^{137} \mathrm{Cs}$ and ${ }^{60} \mathrm{Co}$ gamma sources, which energies are $662 \mathrm{keV}$ and 1170/1333 keV, respectively. Figure 2 shows the plots of observed detection efficiency as the function of the incident gamma-ray energy.

However, there are only 4 data, it is confirmed that the threshold energy changes by using the medium of a different refractive index.

\section{CONCLUSIONS}

In the preliminary experiment, we have verified that the proposed detector has the threshold energy that we can control by changing the refractive index of the radiator. Precise modeling of detector and the deconvolution procedure are required for detail discussion

\section{REFERENCES}

S. Yanagihara, et al., 1993, JSME International Journal B, 36, 6, p.493.

M. Hayashi, et al, 2008, Reactor Dosimetry State of the Art 2008: Proceedings of the 13th International

Symposium, p. 187.

Chamberlain, et al, 1995, Phys. Rev. 100, p. 947. detector which radiator was consisted of distilled water and 


\section{A CHERENKOV THRESHOLD COUNTER USING LIQUID CORE FIBER FOR ESTIMATION OF RADIOACTIVE WASTE INVENTORY}

\author{
Jun Kawarabayashi \\ Nagoya University, Furo- \\ cho, Chikusa-ku, \\ Nagoya,464-8603 Japan \\ Phone : $+81-52-789-4695$, \\ Fax : $+81-52-789-5127$ \\ kawarabayashi@nucl.nagoya- \\ u.ac.jp
}

\author{
Yosuke Sato \\ Nagoya University, Furo- \\ cho, Chikusa-ku, \\ Nagoya,464-8603 Japan \\ Phone : +81-52-789-3790, Fax : \\ $+81-52-789-5127$ \\ Satou.yousuke@f.mbox.nagoya- \\ u.ac.jp
}

\author{
Hikaru Hayakawa \\ Nagoya University, Furo-cho, \\ Chikusa-ku, Nagoya,464-8603 \\ Japan \\ Phone : +81-52-789-3790, Fax : \\ $+81-52-789-5127$ \\ Hayakawa.hikaru@d.mbox.nagoya- \\ u.ac.jp
}

\section{Hideki Tomita}

Nagoya University, Furo-cho, Chikusa-ku, Nagoya,464-8603 Japan

Phone : +81-52-789-4695, Fax : +81-52-789-

5127

tomita@nagoya-u.jp

\author{
Tetsuo Iguchi \\ Nagoya University, Furo-cho, Chikusa-ku, \\ Nagoya,464-8603 Japan \\ Phone : +81-52-789-4680, Fax : +81-52-789-5127 \\ t-iguchi@nucl.nagoya-u.ac.jp
}

Keywords: low level waste, Cherenkov counter, liquid core fiber, decommissioning, radiation robust

\begin{abstract}
Decommissioning of nuclear power plant will increase a number of radioactive wastes near future. In Japan, some of the radioactive wastes will show high intensity of radiation and must be disposed in underground that deep enough from human activities. Therefore, it is important to estimate the inventory of such intense radioactive wastes. But it is difficult to verify the inventory of such radioactive wastes directly by a radiation counter because its intensity is too high to estimate by conventional pulse counting method

In order to verify the inventory of such radioactive wastes directly, we propose a threshold detector with liquid core fiber as a Cherenkov counter which is applicable to high intensity radiation field. As a result of the preliminary experiment using ${ }^{137} \mathrm{Cs}$ and ${ }^{60} \mathrm{Co}$ gamma sources, we have confirmed that the proposal counter worked as the threshold detector. In this paper, we present the principle of this detector and show a preliminary result of detector response to gamma-rays.
\end{abstract}

\section{INTRODUCTION}

Decommissioning of nuclear power plant (Yanagihara 1993) will be important in near future and will increase the amount of radioactive waste. In Japan, the radioactive wastes are classified into two levels, high level waste (HLW) and low level waste (LLW). While the HLW is produced by reprocessing operation of spent nuclear fuel and is going to be buried in a deep permanent repository. On the other hand, the LLW is considered to be all disposable waste created during the operation of nuclear facilities which is not classified as the HLW and discarded in a landfill. A part of the LLW produced by the decommissioning of nuclear power plant, such as a shroud of a reactor core, will show high intensity of radiation and must be disposed in underground that deep enough from human activities. Therefore, it is important to estimate the inventory of such intense LLW. The inventory is expected by the calculation (Grudzevich 2002) of neutron transport, fuel burn-up and activation data with the plant operation history. The trace elements in structural material play a major role in these processes (Stephens 1978) and uncertainties of these materials require a big margin when disposing of this LLW. But it is difficult to verify the inventory of such LLW directly by a radiation counter because its intensity is too high to estimate by conventional pulse counting method.

In order to verify the inventory of such LLW directly, we propose a threshold detector with liquid core fiber (Hayashi 2008) as a Cherenkov counter (Chamberlain 1995) which is applicable to high intensity radiation field. In this paper, we 


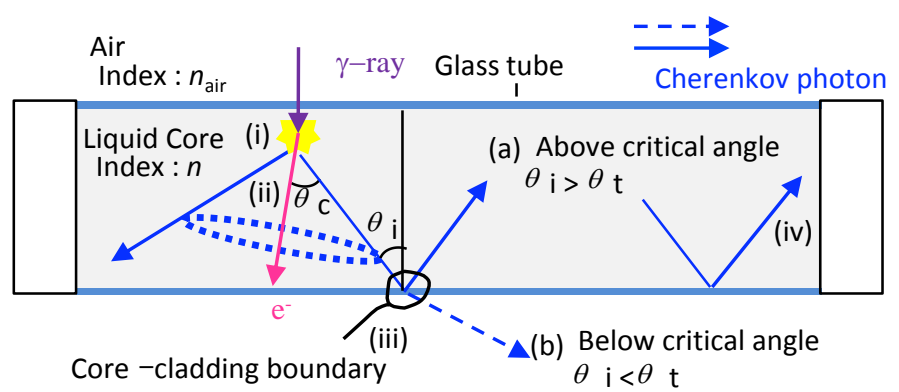

Fig. 1. Schematic view of proposed detector.

(i)Photoelectric interaction (ii) Emission of Cherenkov photons (low energy $\gamma$-ray can't emit photons) . (iii) At coreclad boundary: (a) The photon is reflected, if the angle $\theta_{i}$ is larger than the critical angle $\theta_{t}$. (b) The photon is refracted, if the angle $\theta_{i}$ is smaller than the critical angle $\theta_{t}$. (iv) Only reflecting photons are reached at the PMT. (v)Acquisition of time-of-flight signals from two PMT

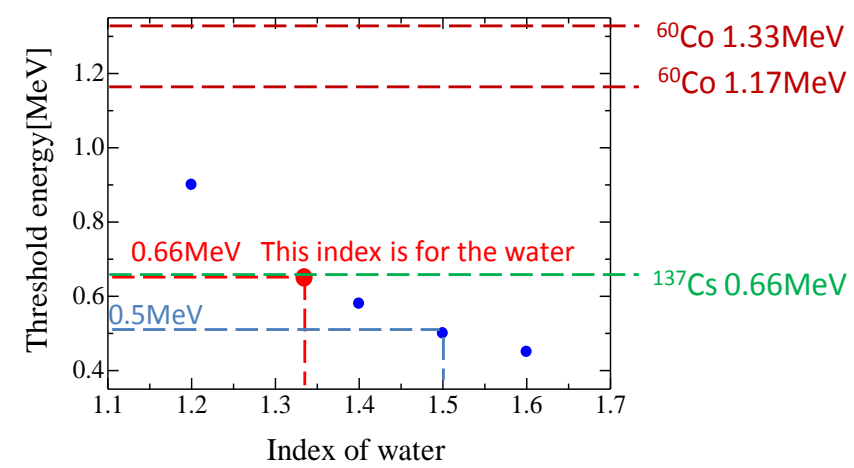

Fig. 2. The threshold energy v.s. refractive index when the core is aqueous solution. The red dot shows the refractive index 1.334 of the pure water.

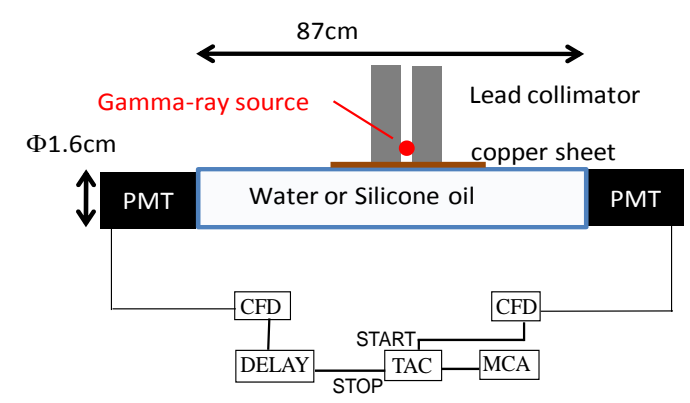

Fig. 3. Experiment setup. Copper plate was placed between the Cherenkov radiator and collimated gamma-ray to shield beta particles from radiation source.

present the principle of this detector and show a preliminary result of detector response to gamma-rays.

\section{PRINCIPLE OF DETECTOR}

In order to estimate the inventory of the LLW, it is important to control the detector to have no efficiency to low energy gamma-ray. Because the LLW is going to be packed in a sturdy container that generates a lot of scattered secondary gamma-rays which energy are lower than the original ones.

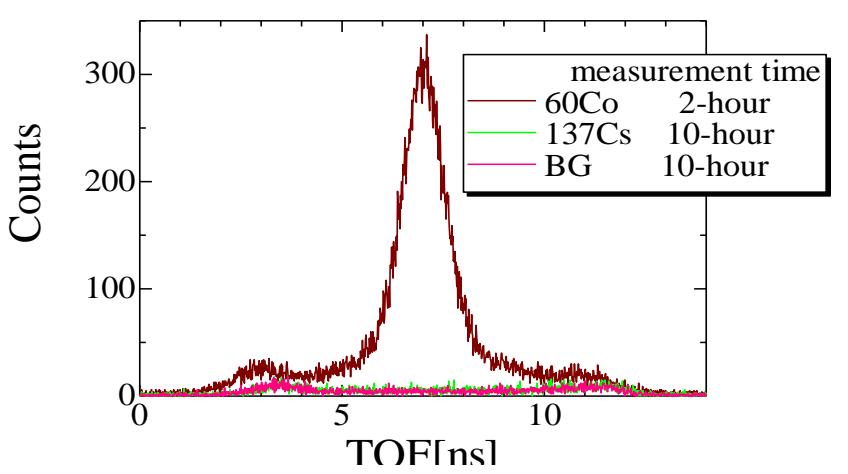

Fig. 4. Detector response to ${ }^{60} \mathrm{Co}(350 \mathrm{kBq})$ [1.33MeV, $1.17 \mathrm{MeV}]$ or ${ }^{137} \mathrm{Cs}(33 \mathrm{kBq}) \quad[0.66 \mathrm{MeV}]$ and background when radiator is consisted of water.

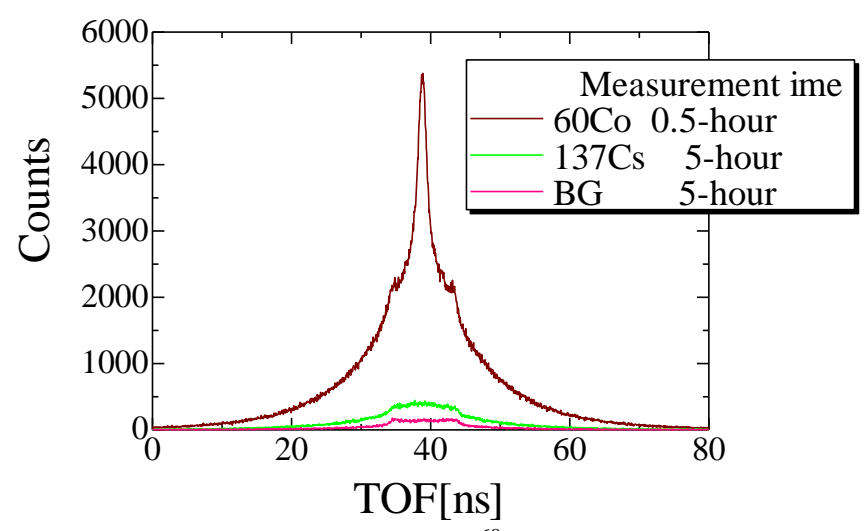

Fig. 5. Detector response to ${ }^{60} \mathrm{Co}(350 \mathrm{kBq})$ [1.33MeV, 1.17 MeV], ${ }^{137} \mathrm{Cs}(33 \mathrm{kBq}) \quad[0.66 \mathrm{MeV}]$ and background when radiator is consisted of silicone oil.

$\left[\times 10^{-4}\right]$

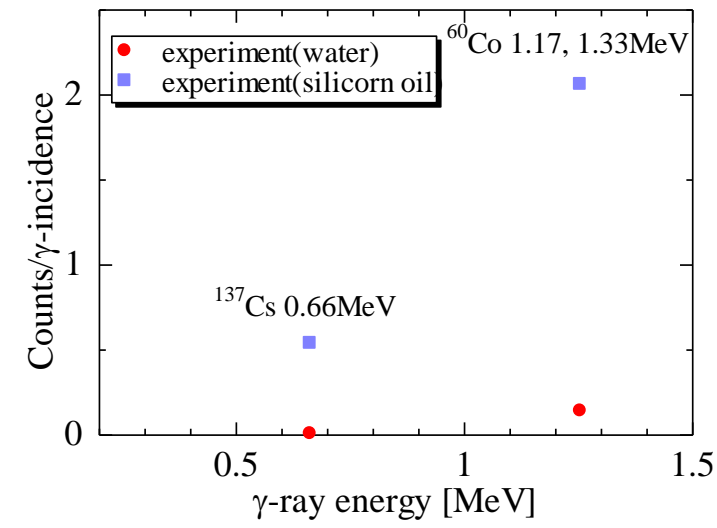

Fig. 6. Detection efficiencies for two radioactive material.

Figure 1 shows a schematic view of this detector. The radiator which consists of fluid is irradiated by the collimated gamma-rays from the LLW that generate photo-electrons which emit Cherenkov photons (Cherenkov 1937) in the direction of $\theta_{c}$. This angle of $\theta_{c}$ is derived from refractive index $n$ of the radiator and the kinetic energy of photoelectron as follows,

$$
\cos \theta_{c}=c / n v_{e},
$$

where $c$ and $v_{e}$ are the speed of light and the photo-electron respectively. Only the Cherenkov photons can be reached at photomultiplier (PMT) located at either end of the radiator if 
the incident angle $\theta_{i}$ is larger than the value of $\theta_{t}$ which is decided by a critical angle of total internal reflection as follows,

$$
\sin \theta_{t}=n_{\text {air }} / n .
$$

From these equations, if the energy of incident gamma-ray increases, the probability that these Cherenkov photons arrive at either end of the radiator increases. This fact shows that if two PMT work in coincidence, this detector works as a threshold counter which has sensitivity only to high energy gamma-ray and the threshold energy can be controlled by the index of the radiator.

If the threshold energy is set to the one of target gamma-ray energy ( i.e. ${ }^{60} \mathrm{Co}$ or ${ }^{108 \mathrm{~m}} \mathrm{Ag}$ ), the gamma-ray which is scattered by surrounding material cannot produce any response in the detector, because the energy of the scattered gamma-ray is lower than that of the original gamma-ray. Arranging these detectors around the LLW with several kinds of adequate refractive indexes, the differences between them show the inventory of the LLW and are suitable for verifying the inventory calculation.

\section{CALCULATED RESPONSE TO GAMMA-RAY}

To evaluate the detector response to gamma-ray, the probability that a pair of Cherenkov photons reached to both PMTs was estimated by a Monte Carlo method with EGS5 (electron gamma shower) code (Hirayama 2010) and original photon transport calculation. The parameters used in the calculation are the following. The core length is $87 \mathrm{~cm}$ with cylindrical form $(\phi 1.6 \mathrm{~cm})$. The outside refractive index is 1.0 . The quantum efficiency of the PMT we used is $25 \%$. And figure 2 shows the threshold energy v.s. the refractive index when the core is aqueous solution. As a result, it was confirmed that the value of the threshold energy decreased according to an increase in the refractive index of the core water.

\section{EXPERIMENTAL RESPONSE TO GAMMA-RAYS}

To confirm the detector response to gamma-ray, preliminary experiment was carried out with a proposed detector which radiator was consisted of distilled water and silicone oil (KF-56 Shin-Etsu Chemical Co., Ltd.) covered by a pyrex glass tube. The center position of the detector was irradiated by gamma-rays from ${ }^{137} \mathrm{Cs}$ and ${ }^{60} \mathrm{Co}$ gamma sources, which energy are $662 \mathrm{keV}$ and 1170/1333 keV, respectively. The experimental setup is shown in Figure 3. The time interval of the Cherenkov photons at either end of the radiator was measured with a conventional Time-of-Flight (TOF) method.

Figure 4 shows the time spectrums of this detector in the case with ${ }^{60} \mathrm{Co},{ }^{137} \mathrm{Cs}$ and no gamma source respectively when the radiator is consisted of water. Two smaller peaks were obtained at the end positions of the radiator in both cases corresponding to the light reflection of PMT's entrance window. A clear peak corresponding to the incident gammaray from ${ }^{60} \mathrm{Co}$ gamma sources was observed at the center of the spectrum, while there is no peak at the center by the irradiation of gamma-rays from ${ }^{137} \mathrm{Cs}$. Figure 5 shows also the time spectrum when silicone oil is used. There are peaks not only for ${ }^{60} \mathrm{Co}$ but also for ${ }^{137} \mathrm{Cs}$. Subtracting the background spectrum from foreground of both results (water and silicone oil), the detection efficiencies was obtained. Figure 6 shows the plots of the experimental results of the detection efficiency as the function of the incident gamma-ray energy.

However, there are only 4 data, it is confirmed that threshold energy changes by using the medium of a different refractive index as shown in the Figure 2. And it has been verified the detector has the threshold energy controlled by the value of the refractive index. More precise modeling of detector, such as attenuation of light as a function of wave length, is required for more detail discussion.

\section{CONCLUSIONS}

For the verifying the inventory of the high intensity LLW, we have proposed a Cherenkov counter using a liquid radiator as a radiation robust detector. In the preliminary experiment, we have obtained the detection efficiency of proposed detector which threshold energy agreed well to the expectation by a Monte Carlo calculation and have verified the detector has the threshold energy that we can control by changing the refractive index. For future works, precise modeling of detector and the deconvolution procedure are required for detail discussion.

\section{REFERENCES}

S. Yanagihara, et al., 1993, JSME International Journal B, 36, 6, p.493.

O. Grudzevich, et al., 2002, J. Nucl. Sci. Tech, Suppl.2, p.1437.

J. J. Stephens Jr. and R. O. Pohl, 1978, Nucl.Eng. Design 47, p. 125 .

M. Hayashi, et al, 2008, Reactor Dosimetry State of the Art 2008: Proceedings of the 13th International Symposium, p. 187.

Chamberlain, et al, 1995, Phys. Rev. 100, p. 947.

P.A. Cherenkov, 1937, Phys. Rev. 52, p. 378.

H. Hirayama, et al., 2010, The EGS5 Code System, SLAC

Report. SLAC-R-730, KEK Report. 2005-8. 